Document downloaded from:

http://hdl.handle.net/10251/142281

This paper must be cited as:

Ortiz-Mayordomo, S.; Tavares De Araujo Cesariny Calafate, CM.; Cano, J.; Manzoni, P.; Toh, C. (06-0). A UAV-Based Content Delivery Architecture for Rural Areas and Future Smart Cities. IEEE Internet Computing. 23(1):29-36. https://doi.org/10.1109/MIC.2018.2884277

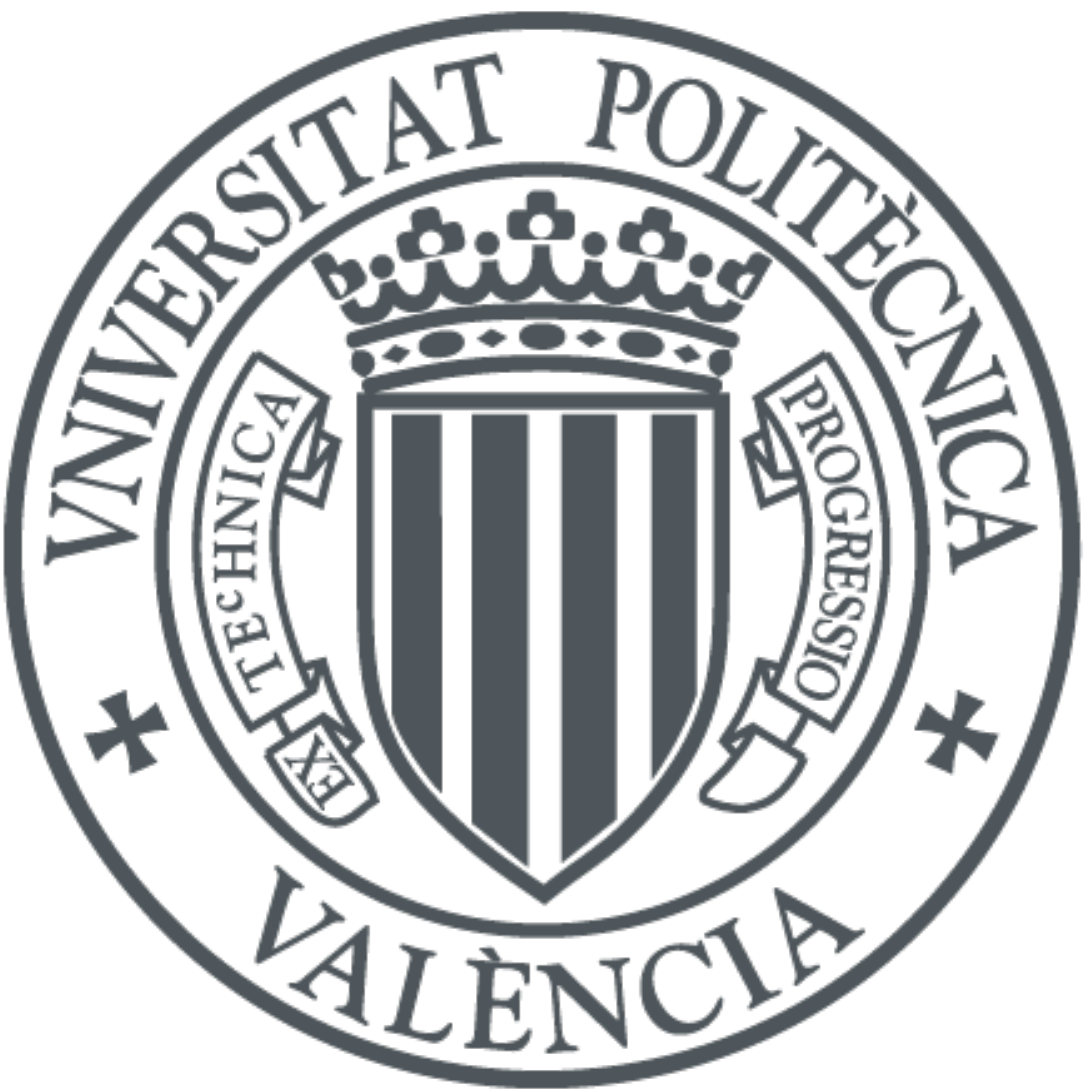

The final publication is available at

https://doi.org/10.1109/MIC.2018.2884277

Copyright Institute of Electrical and Electronics Engineers

Additional Information 


\section{An UAV-based Content Delivery Architecture for Rural Areas and Future Smart Cities}

Sergio Ortiz,

Universitat Politècnica de

València, Spain

Carlos T. Calafate

Universitat Politècnica de Valènciam, Spain

Chai K. Toh

National Tsing Hua

University (NTHU), Taiwan

Juan-Carlos Cano

Universitat Politècnica de

València, Spain

Pietro Manzoni

Universitat Politècnica de

València, Spain
Content delivery in vehicular environments can serve multiple purposes, such as safety, entertainment and news delivery that can be geographically relevant to vehicles traveling within a certain area. The traditional approach to address this problem, based on fixed networking infrastructure, suffers from two drawbacks: (i) the efficient delivery of large-sized contents to multiple moving receivers simultaneously can be hard to achieve; and (ii) most of the roads outside the main urban areas lack such fixed infrastructures due to economic reasons. In this paper, we tackle both these issues by proposing rapidly deployable wireless access infrastructures combining RaptorQ-protected

content diffusion and Unmanned Aerial Vehicles (UAVs). We performed experiments using actual vehicles and UAVs, and our results showed that: (i) RaptorQ-based content dissemination mechanisms is highly efficient when transmitting to multiple moving receivers simultaneously, and (ii) UAVs can serve as cheap, effective, and rapidly deployable mobile wireless access elements. 


\section{INTRODUCTION}

Vehicular networks are receiving much attention recently due to their expected growth in the coming years. By equipping vehicles with on-board units (OBUs) acting as network nodes ${ }^{1}$, these connected vehicles can address new challenges related to both safety and infotainment. In these environments, communication issues arise due to the high number of networks nodes involved, and due to signal obstruction caused by buildings and other urban artifacts ${ }^{2}$.

Unmanned Aerial Vehicles (UAVs), or drones, have been used by Facebook ${ }^{3}$ in their Acquila project. UAVs are semi-autonomous or fully autonomous unmanned aircrafts that have embedded sensors, cameras and communication equipment. Originally, UAVs were deployed for military applications. Nowadays, their use includes recreation, advertising, industry, communications, border surveillance, disaster response, fire fighting, and transportation of medicines or first aid ${ }^{4}$. Currently, the main challenges associated with UAV deployment are mainly flight autonomy, maintaining stable UAV-to-Ground station communication links, obstacle avoidance, and swarm formation ${ }^{5}$.

Combining UAVs and vehicles emerges as an interesting and novel topic with multiple applications, including sending warnings to drivers about unexpected danger or even regulating traffic. This can be especially useful in catastrophic areas, or in areas with minimal or null cellular coverage, where the strategical placement of a few UAVs can quickly reverse the situation. Hence, there is a need to investigate content delivery scheme for vehicular networks using UAV-based wireless access. This forms the main motivation of our work.

The rest of this paper is organized as follows: in the next section we shall present some related works on content delivery, drones, and Raptor codes. We then proceed to describe our proposed architecture. Thereafter, we present the relevant implementation details, followed by experimental results. Finally, we present the main conclusions of our paper.

\section{RELATED WORKS}

Our work spans across three different research areas: (i) vehicular networking, (ii) UAVs in vehicular networks, and (iii) Raptor codes. For each of these areas, significant contributions can be found in the literature.

Regarding vehicular networking, there are a vast range of works addressing different topics that include routing and MAC protocols, content delivery, efficient dissemination of warning messages, trust management, and infotainment, among others ${ }^{6}$.

Concerning UAVs, many papers have proposed using UAVs to improve wireless communications. However, only few have combined UAVs with vehicular networks. VDNet ${ }^{7}$ proposed a routing protocol for vehicle-to-vehicle (V2V) communications based on UAVs to decrease the average end-to-end packet delivery delay. To make this possible, some vehicles in VDNet are equipped with an on-board UAV which can deliver data messages directly to the destination, relay messages in a multi-hop wireless route, and collect location information while flying above road traffic.

Seliem et al. ${ }^{8}$ proposed a mathematical framework to determine the minimum UAV density (maximum separation distance between two adjacent UAVs) that stochastically limits the worst case for the vehicle-to-UAV packet delivery delay. In addition, they proposed a drones-active service (DAS) that is added to the location service in a VANET to obtain the required number of active UAVs based on the current vehicular density, while satisfying a probabilistic requirement for vehicle-to-UAV packet delivery delay.

Shi et al. ${ }^{9}$ proposed DAVN, a solution that efficiently integrates the communication and networking technologies of drones and connected vehicles. The authors showed that drones can improve vehicle-to-vehicle connectivity, infrastructure coverage, network information collection ability, and network interworking efficiency. Similarly, Oubbati et al. ${ }^{10}$ studied how UAVs operating in ad-hoc mode can cooperate with VANET on the ground so as to assist in the routing process, and improve the reliability of the data delivery by bridging the communication gap whenever possible. 
Finally, concerning Raptor codes ${ }^{11}$, they are one of the most efficient error correction techniques (FEC). They are used to control the errors in the transmission of data over unreliable and noisy communication channels. Many researchers have worked on Raptor codes. For instance, Todor Mladenov et al. ${ }^{12}$ make a comparison between Raptor and RaptorQ in terms of encoding properties, decoding performance, and energy efficiency in an embedded system, concluding that RaptorQ's encoding performance is better, but it consumes more power than legacy Raptor encoding.

Our paper differs from previous works because our solution is unique in the sense that we combined broadcast-based content delivery with the forward error correction scheme known as RaptorQ. Furthermore, we assess the impact of different infrastructure modes of operation, smartphone models, and we measure the range enhancements achieved using UAVs when flying at different altitudes.

\section{PROPOSED ARCHITECTURE}

In this section, we describe our proposed architecture, including our resilient content delivery solution, implementation details, deployment issues, and infrastructure elements.

The envisioned target scenario is one where an infrastructure element is constantly broadcasting some specific content, which can be any sort of multimedia file(s), to vehicles passing by. This content is encoded using RaptorQ. In a previous study ${ }^{13}$ it was shown that RaptorQ is indeed a very efficient FEC scheme, and allows a potentially limitless sequence of encoding symbols to be generated. Within vehicles, the content is received by the driver's smartphone, and the multimedia content is then decoded and played back automatically, without requiring user intervention. Examples of the applicability of our solution includes: (a) location-based security warnings, and (b) touristic advertisements.

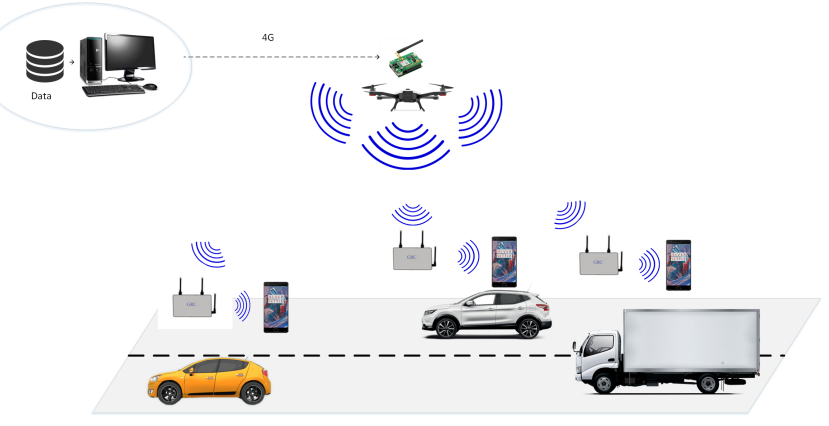

Figure 1. Drone-based deployment approach.

Our work differs from the standard approach in that we do not rely on any static infrastructure. Instead, we have a mobile infrastructure element (see Figure 1). We equip Unmanned Aerial Vehicles (UAVs) of the multirotor type with our Raspberry Pi-based broadcasting unit (see Fig. 2), so that they can become highly flexible and mobile infrastructure elements. The UAVs rely on the ad-hoc wireless networking paradigm to connect to vehicles using the $5.8 \mathrm{GHz}$ band. Since most smartphones do not support this mode of operation, each vehicle is equipped with an on-board unit offering ad-hoc communications at this frequency band. The on-board unit acts as an information relay, conveying the received information to the smartphone on the vehicles by creating a WiFi hotspot in the $2.4 \mathrm{GHz}$ band. The smartphone will receive the RaptorQ-encoded information, playing it back upon successful decoding.

In our work, UAVs will be used to perform content broadcasting at different altitudes, allowing us to assess the improvements that can be achieved in terms of content delivery efficiency in the presence of irregular terrains. In terms of autonomy, our UAVs can hover at a fixed position for a typical time of up to 30 minutes, similarly to most UAVs in the market. Note that this value can be extended to beyond 2 hours if hybrid multicopters are used (e.g. Quaternium Hybrix.20 UAV, see http://www.quaternium.com/uav/hybrix-20/). 


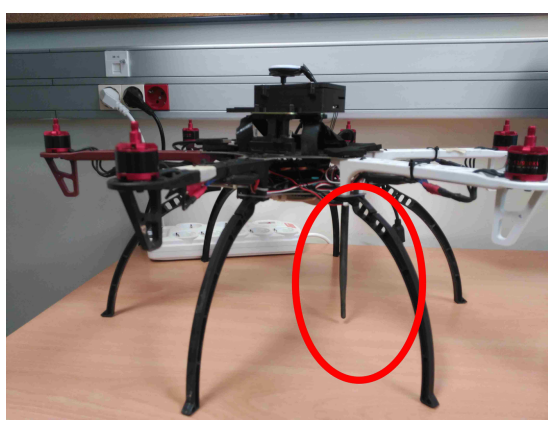

Figure 2. Self-designed Hexacopter.

\section{IMPLEMENTATION DETAILS}

To assess the performance achieved by our mobile Road-side Unit solution in real-life situations, we relied on a Raspberry Pi embedded with an IEEE 802.11a adapter from ALFA Network, model AWUS051NH V2, which has an output power of $25 \mathrm{dBm}$ and a sensitivity of $-93 \mathrm{dBm}$. The RaptorQ server is also running at the Raspberry Pi, making it an all-in-one solution that is cheap, energy-efficient, and easy to deploy due to its small size. In terms of software, the server application was developed in Java, and it first encodes the target file(s) using RaptorQ, and then it proceeds by multicasting this information in an infinite loop, so that all passing-by vehicles become potential receivers, making it a fully scalable solution.

Regarding client devices, we relied on Android terminals for testing. These terminals operate on both 2.4 and $5.8 \mathrm{GHz}$ bands. In addition, they run a RaptorQ-enabled application capable of decoding RaptorQ-encoded messages. In our tests, this application is also able to log the GPS coodinates of the receiver upon receiving a data packet, along with the corresponding timestamp, as well as the time instant at which the content was decoded, thus enabling an in-depth performance evaluation following our tests.

Concerning the vehicle on-board units adopted, they are also based on Raspberry Pi devices. In particular, such a solution is denoted as GRCBox ${ }^{14}$. It provides great flexibility by creating a WiFi hotspot within the vehicles, thus allowing smartphones to dynamically connect to a variety of external technologies, depending on the type of application used.

\section{EXPERIMENTAL RESULTS}

In urban scenarios, where street length is relatively short, and where the presence of tall buildings impede communications to other nearby streets, the static infrastructure approach seems to be an adequate solution. In a previous work ${ }^{15}$, we had studied this problem, evaluating the performance of our architecture when deploying static RSUs. However, when content broadcasting takes place outside urban areas, in the main roads or even highways, this static setting may experience some limitations. In fact, since the vehicle speed becomes much higher, the available time to deliver the target contents to vehicles is reduced. Thus, any solution that helps at increasing the amount of data delivered is desirable. A typical solution could be adopting higher gain antennas for broadcasting. However, in the presence of irregular terrain, the extra gain can become unrealizable if line-of-sight conditions are limited to small distances from the broadcasting antenna. Thus, our approach in this paper is to adopt UAVs, so that the broadcasting can be done at high altitudes, thereby extending the line of sight between the transmitter and potential receivers.

With these issues in mind, in this section, we present and discuss the results obtained in our tests, all carried out in a real outdoor environment. Specifically, we will show the advantages of having the broadcaster located at high altitudes compared to standard conditions, where the broadcasting antenna is only slightly above the road level.

Taking the above-mentioned considerations into account, we equipped an UAV with our Raspberry Pi-based broadcasting unit embedded with a $5 \mathrm{dBi}$ antenna, and operating in the ad-hoc mode 
(RSU), as shown in figure $\backslash$ ref $\{$ dronver\}. Tests were performed in the municipality of Casinos, near Valencia, Spain. There, we picked a road with an irregular terrain profile for our tests. In particular, the road segment selected for our tests has an overall length of $10 \mathrm{~km}$, and the speed limit is at $100 \mathrm{~km} / \mathrm{h}$. The transmitter is located near the middle of the road segment, while the vehicle used for testing, and acting as the receiver in our tests, was travelling near the speed limit at all times.

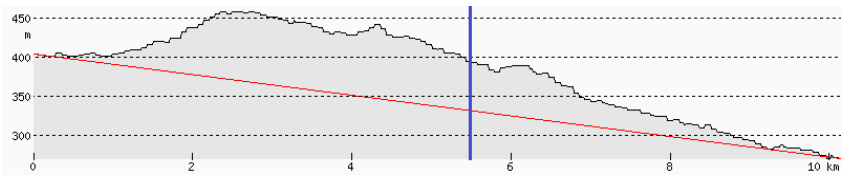

Figure 3. Terrain profile.

Figure 3 illustrates the terrain profile along the road segment selected for our tests. The vertical blue line indicates the UAV position. We can observe that there is a difference of about 450 meters between the highest road elevation in comparison to the lowest.

Regarding the characteristics of the contents to be broadcasted, these consist of a single image of size 350 kilobytes, and the inter-packet interval was set to $10 \mathrm{~ms}$. The broadcasting data rate for this setting is $800 \mathrm{kbit} / \mathrm{s}$.

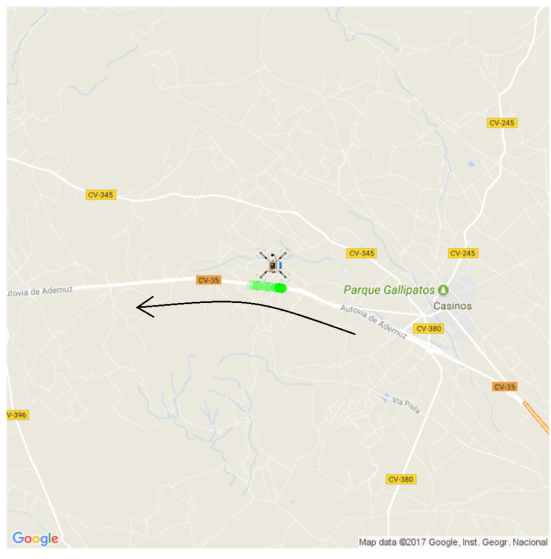

a) $5 \mathrm{~m}$

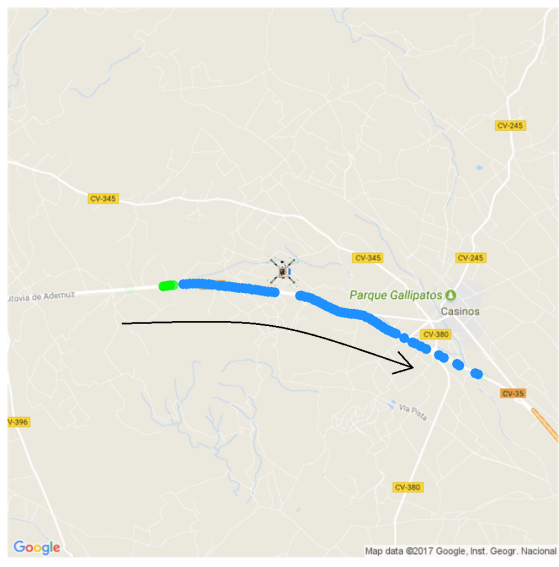

c) $60 \mathrm{~m}$

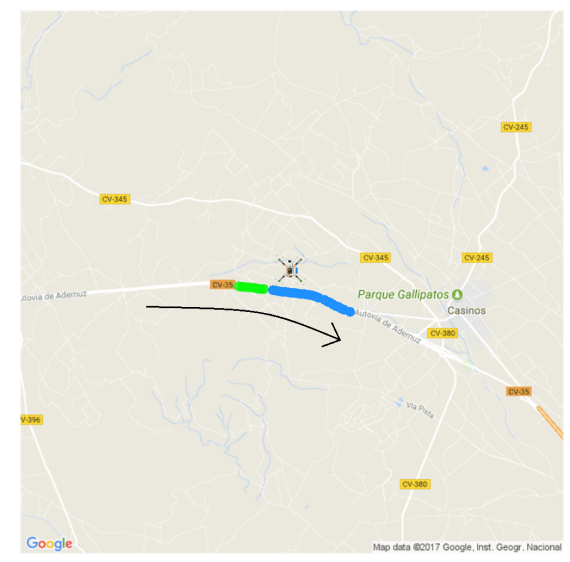

b) $25 \mathrm{~m}$

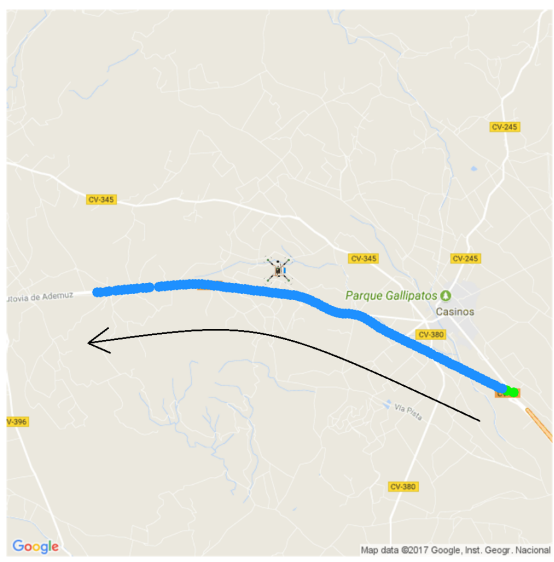

d) $90 \mathrm{~m}$

Figure 4. Content reception effectiveness at different UAV altitudes. 
In figure 4, we can see that there is a great difference in terms of data reception effectiveness for different UAV flight altitudes. At 5 meters, the situation represents a static infrastructure deployment, some data is received, but it is not enough even to decode a content with such a small size such as the one we have used. We started to benefit from the increased altitudes in the UAV, as the content reception region increases considerably with altitude. The best situation for our tests is achieved at 90 meters, since all the data required for decoding are received immediately after our tests have started, and during the rest of the path the incoming data can be discarded.

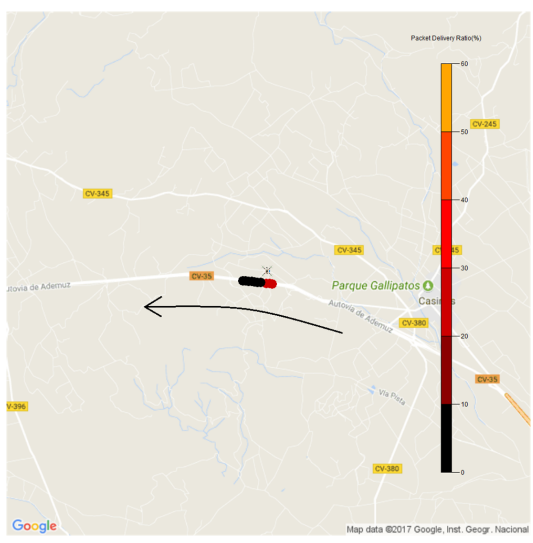

a) $5 \mathrm{~m}$

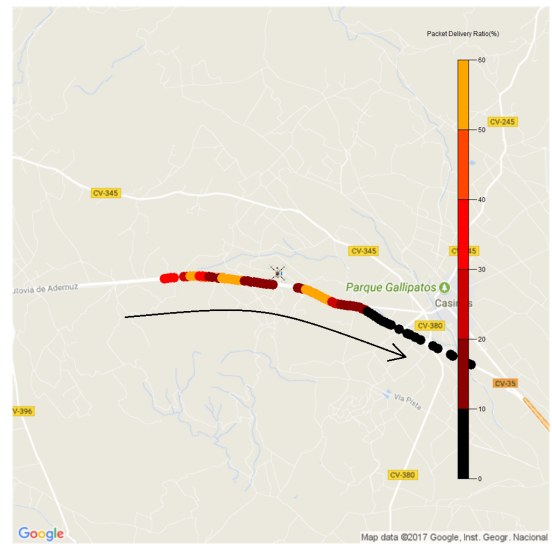

c) $60 \mathrm{~m}$

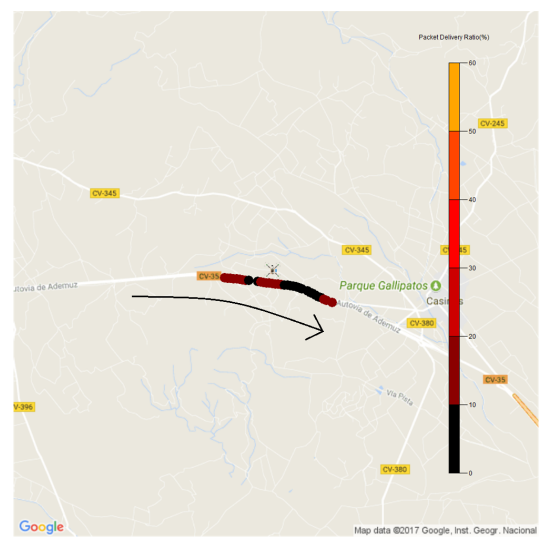

b) $25 \mathrm{~m}$

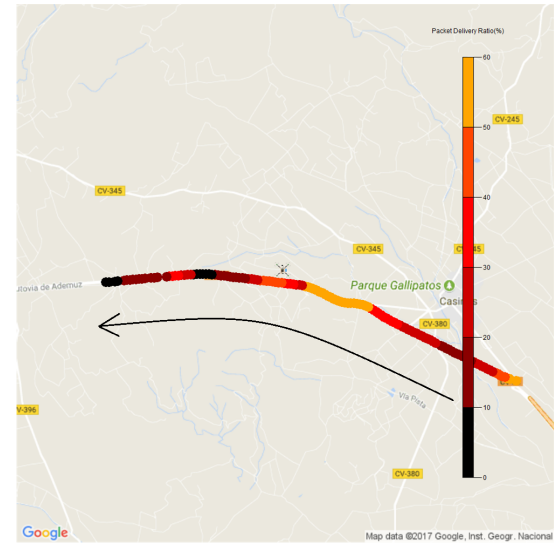

d) $90 \mathrm{~m}$

Fig. 5. Delivery ratio at different UAV altitudes.

Regarding the delivery ratio, figure 5 shows that such ratio increases as the vehicles approaches the UAV coordinates, and then it gradually decreases again as it moves past it. This is especially noticeable for the 90 meters altitude scenario, since before reaching the UAV coordinates the delivery ratio increases from $20 \%$ to $60 \%$, and once it passes the UAV and moves away, the delivery ratio drops to values between $5 \%$ to $15 \%$. This shows that there is some Doppler effect associated to the vehicle's speed, which negatively affects communications when the vehicle is moving away from the transmitter, as compared to the situation where it moves towards the transmitter.

From Table 1, we can see the total amount of data received, the time span during which packet reception events took place, and the total coverage distance of the transmitter, in meters, for the different UAV altitudes tested. It now becomes clear that increasing the transmitting altitude can be highly beneficial for content delivery, and being that at high altitudes it becomes possible to deliver contents of greater sizes, and deliver them with a greater reliability, due to the extended coverage and time span. In particular, comparing the worst situation ( 5 meters, similar to static infrastructure deployments) with the best situation ( 90 meters), the overall data delivered increases 
from $0.2 \mathrm{MB}$ to $10.26 \mathrm{MB}$, a factor of more than 50 times, which is indeed a very significant and substantial improvement.

Table 1. Data, time and distance for different altitudes.

\begin{tabular}{|c|c|c|c|c|}
\hline Altitude (m) & Data (MB) & Time (s) & $\begin{array}{c}\text { Distance } \\
(\boldsymbol{m})\end{array}$ & $\begin{array}{c}\text { Data rate } \\
\text { (kbit/s) }\end{array}$ \\
\hline 5 & 0.2 & 21.68 & 550 & 9,7 \\
\hline 25 & 0.78 & 74.12 & 2000 & 11,0 \\
\hline 60 & 5.11 & 231.2 & 6300 & 23,2 \\
\hline 90 & 10.26 & 285.81 & 7600 & 37,64 \\
\hline
\end{tabular}

Overall, these results clearly point out the benefits of using UAVs as mobile infrastructure elements in roads or highways lacking static infrastructures. It can also be used for on-demand content broadcast for advertisements and road safety. In fact, their quick deployment can help at mitigating unexpected road problems. Future smart cities will need such on-demand and rapidly-deployable content-as-a-service for greater flexibility and robust content delivery.

\section{CONCLUSIONS}

In this paper, we have studied the performance of content delivery in vehicular networks. To this aim, we have developed a drone-based solution that adopts a FEC scheme based on RaptorQ, which ensures that the data to be transmitted will be received correctly on all devices, even in the presence of channel losses, thereby achieving seamless data integrity.

Tests performed in a road environment with irregular terrains and high vehicular speeds (100 $\mathrm{km} / \mathrm{h}$ ) show that having a mobile infrastructure deployment based on UAVs can improve content delivery effectiveness compared to static infrastructure deployments since it becomes possible to locate the transmitter at a high altitude (90 meters), a situation that is able to substantially increase the coverage range, and a factor of 50 on the size of the contents that can be delivered successfully.

\section{REFERENCES}

[1] S. Youse, M.S. Mousavi, and M. Fathy. Vehicular ad hoc networks (VANETs): challenges and perspectives. In ITS Telecommunications Proceedings, 2006 6th International Conference on, pages 761-766. IEEE, 2006.

[2] S.A. Hadiwardoyo, A. Tomás, E. Hernandez-Orallo, C.T. Calafate, J.C. Cano, P. Manzoni. Empirical Study and Modeling of Vehicular Communications at Intersections in the $5 \mathrm{GHz}$ Band, Mobile Information Systems, special issue on Connected Vehicles: Applications and Communication Challenges (CVAC), Volume 2017, Article ID 2861827.

[3] M. Zuckerberg. The technology behind aquila. https://www.facebook.com/notes/mark-zuckerberg/the-technology-behind-aquila/10153916136506634/ (Accessed: September 24, 2018), 2016.

[4] US Department of Transportation, "Unmanned Aircraft System (UAS) Service Demand 20152035: Literature Review \& Projections of Future Usage," tech. rep., v.0.1, DOT-VNTSC-DoD-1301, Sept. 2013.

[5] L. Gupta, R. Jain and G. Vaszkun, "Survey of Important Issues in UAV Communication Networks," in IEEE Communications Surveys \& Tutorials, vol. 18, no. 2, pp. 1123-1152, Secondquarter 2016.

[6] H. Hartenstein and L. P. Laberteaux, "A tutorial survey on vehicular ad hoc networks," in IEEE Communications Magazine, vol. 46, no. 6, pp. 164-171, June 2008. 
[7] X. Wang, L. Fu, Y. Zhang, X. Gan, and X. Wang. Vdnet: an infrastructure-less uav-assisted sparse vanet system with vehicle location prediction. Wireless Communications and Mobile Computing, 16(17):2991-3003, 2016.

[8] H. Seliem, M. H. Ahmed, R. Shahidi and M. S. Shehata, "Delay analysis for drone-based vehicular Ad-Hoc Networks," 2017 IEEE 28th Annual International Symposium on Personal, Indoor, and Mobile Radio Communications (PIMRC), Montreal, QC, 2017, pp. 1-7.

[9] W. Shi, H. Zhou, J. Li, W. Xu, N. Zhang and X. Shen, "Drone Assisted Vehicular Networks: Architecture, Challenges and Opportunities," in IEEE Network, vol. 32, no. 3, pp. 130-137, May/June 2018.

[10] O.S. Oubbati, A. Lakas, F. Zhou, M. Güneş, N. Lagraa, M.b. Yagoubi, Intelligent UAV-assisted routing protocol for urban VANETs, Computer Communications, Volume 107,

2017, Pages 93-111.

[11] Amin Shokrollahi, "Raptor Codes," IEEE Transactions on Information Theory, vol. 52, pp. 2551-2567, 2006.

[12] T. Mladenov, K. Kim, and S. Nooshabadi. Forward error correction with RaptorQ code on embedded systems. In Circuits and Systems (MWSCAS), IEEE 54th International Midwest Symposium on, pages 1-4, 2011.

[13] C.T. Calafate, G. Fortino, S. Fritsch, J. Monteiro; J.C. Cano, P. Manzoni. "An efficient and robust content delivery solution for IEEE $802.11 \mathrm{p}$ vehicular environments", Journal of Network and Computer Applications, Volume: 35, Issue:2, pages 753-762. March 2012.

[14] S.M. Tornell, S. Patra, C.T. Calafate, J.C. Cano, and P. Manzoni. Grcbox: extending smartphone connectivity in vehicular networks. International Journal of Distributed Sensor Networks, 2015.

[15] S. Ortiz, C.T. Calafate, J.C. Cano, P. Manzoni, Evaluating RaptorQ-based content broadcasting strategies in vehicular environments, IEEE 87th Vehicular Technology Conference (VTC2018Spring), Porto, Portugal. June 3-6, 2018.

\section{ABOUT THE AUTHORS}

Sergio Ortiz received a degree in Computer Engineering from Technical University of Valencia, Spain, in 2016. He later received a Master's Degree in Computer and Network Engineering from the same university. In 2017, he carried out several research activities within the Networking Research Group (GRC). His research topics include wireless and vehicular networks, and content protection using Raptor codes. (E-mail: serorma1@disca.upv.es).

Carlos T. Calafate is an associate professor in the Department of Computer Engineering at the Technical University of Valencia (UPV) in Spain. He graduated with honors in Electrical and Computer Engineering at the University of Oporto (Portugal) in 2001. He received his $\mathrm{Ph} . \mathrm{D}$. degree in Informatics from the Technical University of Valencia in 2006, where he has worked since 2002. His research interests include ad-hoc and vehicular networks, UAVs, Smart Cities \& IoT, QoS, network protocols, video streaming, and network security. (E-mail: calafate@disca.upv.es).

Chai K. Toh is currently with National Tsing Hua University. He was Group CTO and Assistant Chief Executive of IDA Singapore, and VP and CTO of Singapore Power Telecom Ltd, co-leading the Singapore's Smart Nation program, IoT, data analytics, smart energy, and smart homes programs. He is an IEEE Fellow (2009) and AAAS Fellow (2009). (E-mail: ck_away@hotmail.com).

Juan-Carlos Cano is a full professor in the Department of Computer Engineering at the Polytechnic University of Valencia (UPV) in Spain. He earned an MSc and a Ph.D. in Computer Science from the UPV in 1994 and 2002 respectively. From 1995-1997 he worked as a programming analyst at IBM's manufacturing division in Valencia. His current research interests include Vehicular Networks, Mobile Ad Hoc Networks, and Pervasive Computing. (E-mail: jucano@disca.upv.es).

Pietro Manzoni received the master's degree in computer science from the Università degli Studi of Milan, Italy, in 1989, and the Ph.D. degree in computer science from the Politecnico di Milano, Italy, in 1995. From 1992 to 1993, he did an internship at the Bellcore Labs, Red 
Bank, NJ, USA, and in 1994, he was a Visiting Researcher with the International Computer Science Institute, Berkeley, CA, USA. He is currently a Full Professor of computer engineering with the Universitat Politècnica de València, Spain. His research activity is related to networking and mobile systems and applied to intelligent transport systems, smart cities and the Internet of Things, and community networks (mesh). He is the Coordinator of the Computer Networks Research Group, Department of Computer Engineering. He is an IEEE Senior Member (2017). (E-mail: pmanzoni@disca.upv.es). 